\title{
Hyperbolic metrics on universal Teichmüller space and extremal problems
}

\author{
SAMUEL L. KRUSHKAL
}

(Presented by M. M. Malamud)

\begin{abstract}
The paper consists of two parts, both related to complex geometry of the universal Teichmüller space. We reprove that all contractible invariant metrics on this space coincide and apply this important fact to solving the general extremal problems for univalent functions with quasiconformal extensions.
\end{abstract}

2010 MSC. 30C75, 30F60, 32G15, 32Q45, 30F45.

Key words and phrases. Quasiconformal, Teichmüller space, Kobayashi metric, Carathéodory metric, Grunsky inequalities, extremal problem.

\section{Introductory remarks}

The complex metric geometry of the universal Teichmüller space causes some interesting phenomena in the classical variational problems for univalent functions. In this paper, we investigate an interaction between these topics.

First we reprove that all contractible invariant metrics on the universal Teichmüller space agree with its intrinsic Teichmüller metric.

Theorem 1.1. The Carathéodory metric of the universal Teichmüller space $\mathbf{T}$ coincides with its Kobayashi metric, hence all invariant metrics on $\mathbf{T}$ are equal the Teichmüller metric.

The infinitesimal forms of these metrics coincide with the canonical Finsler structure on $\mathbf{T}$ which generates the Teichmüller metric. 
This important fact, established in [14] by renormalization of sequences of holomorphic quadratic differentials generating the extremal Beltrami coefficients, underlies various deep applications. Its new proof is much simpler and relies on some results that have intrinsic interest. Note that such a result is still unknown for other Teichmüller spaces.

Theorem 1.1 is deeply connected with general extremal problems and the distortion theorem for univalent functions with quasiconformal extensions and gives rise to their strengthening. We consider these problems in the last section.

\section{Background}

We briefly present here certain results underlying the proof of Theorem 1.1. This exposition is adapted to our special cases. Let $\Delta=\{|z|<$ $1\}, \Delta^{*}=\widehat{\mathbb{C}} \backslash \bar{\Delta}$.

\subsection{Complex geometry of universal Teichmüller space}

The universal Teichmüller space is the space of quasisymmetric homeomorphisms of the unit circle $S^{1}=\partial \Delta$ factorized by Möbius maps. The canonical complex Banach structure on $\mathbf{T}$ is defined by factorization of the ball of the Beltrami coefficients (or complex dilatations)

$$
\operatorname{Belt}(\Delta)_{1}=\left\{\mu \in L_{\infty}(\mathbb{C}): \mu \mid \Delta^{*}=0,\|\mu\|<1\right\},
$$

letting $\mu_{1}, \mu_{2} \in \operatorname{Belt}(\Delta)_{1}$ be equivalent if the corresponding quasiconformal maps $w^{\mu_{1}}, w^{\mu_{2}}$ (solutions to the Beltrami equation $\partial_{\bar{z}} w=\mu \partial_{z} w$ with $\left.\mu=\mu_{1}, \mu_{2}\right)$ coincide on the unit circle $S^{1}=\partial \Delta^{*}$ (hence, on $\overline{\Delta^{*}}$ ). The equivalence classes $\left[w^{\mu}\right]$ are in one-to-one correspondence with the Schwarzian derivatives

$$
S_{w}(z):=\left(\frac{w^{\prime \prime}}{w^{\prime}}\right)^{\prime}-\frac{1}{2}\left(\frac{w^{\prime \prime}}{w^{\prime}}\right)^{2}=\frac{w^{\prime \prime \prime}}{w^{\prime}}-\frac{3}{2}\left(\frac{w^{\prime \prime}}{w^{\prime}}\right)^{2}, \quad w=w^{\mu} \mid \Delta^{*} .
$$

Note that for each locally univalent function $w(z)$ on a simply connected hyperbolic domain $D \subset \widehat{\mathbb{C}}$, its Schwarzian derivative $S_{w}$ belongs to the complex Banach space $\mathbf{B}(D)$ of hyperbolically bounded holomorphic functions on $D$ with the norm

$$
\|\varphi\|_{\mathbf{B}(D)}=\sup _{D} \lambda_{D}^{-2}(z)|\varphi(z)|,
$$

where $\lambda_{D}(z)|d z|$ is the hyperbolic metric on $D$ of Gaussian curvature -4 ; hence $\varphi(z)=O\left(z^{-4}\right)$ as $z \rightarrow \infty$ if $\infty \in D$. In particular, $\lambda_{\Delta}(z)=$ 
$1 /\left(1-|z|^{2}\right)$. The space $\mathbf{B}(D)$ is dual to the Bergman space $A_{1}(D)$, a subspace of $L_{1}(D)$ formed by integrable holomorphic functions on $D$.

The derivatives $S_{w^{\mu}}(z)$ with $\mu \in \operatorname{Belt}(\Delta)_{1}$ range over a bounded domain in the space $\mathbf{B}=\mathbf{B}\left(\Delta^{*}\right)$. This domain models the universal Teichmüller space $\mathbf{T}$, and the defining projection

$$
\phi_{\mathbf{T}}(\mu)=S_{w^{\mu}}: \operatorname{Belt}(\Delta)_{1} \rightarrow \mathbf{T}
$$

is a holomorphic map from $L_{\infty}(\Delta)$ to $\mathbf{B}$. This map is a split submersion, which means that $\phi_{\mathbf{T}}$ has local holomorphic sections (see, e.g., [7]).

The above definition of $\mathbf{T}$ requires a complete normalization of maps $w^{\mu}$, which uniquely defines the values of $w^{\mu}$ on $\Delta^{*}$ by their Schwarzians. We shall use the condition $w^{\mu}(0)=0$ going over from $w^{\mu}$ to the maps

$$
\begin{aligned}
w_{1}^{\mu}(z)=w^{\mu}(z) & -w^{\mu}(0) \\
& =z-\frac{1}{\pi} \iint_{\Delta} \frac{\partial w^{\mu}}{\partial \bar{\zeta}}\left(\frac{1}{\zeta-z}-\frac{1}{\zeta}\right) d \xi d \eta \quad(\zeta=\xi+i \eta),
\end{aligned}
$$

which does not reflect on the Schwarzians. We identify the bounded domain in B filled by $S_{w_{1}^{\mu}}$ with the space $\mathbf{T}$.

The intrinsic Teichmüller metric of the space $\mathbf{T}$ is defined by

$$
\begin{aligned}
\tau_{\mathbf{T}}\left(\phi_{\mathbf{T}}(\mu), \phi_{\mathbf{T}}(\nu)\right) & \\
& =\frac{1}{2} \inf \left\{\log K\left(w^{\mu_{*}} \circ\left(w^{\nu_{*}}\right)^{-1}\right): \mu_{*} \in \phi_{\mathbf{T}}(\mu), \nu_{*} \in \phi_{\mathbf{T}}(\nu)\right\} ;
\end{aligned}
$$

it is generated by the Finsler structure

$$
F_{\mathbf{T}}\left(\phi_{\mathbf{T}}(\mu), \phi_{\mathbf{T}}^{\prime}(\mu) \nu\right)=\inf \left\{\left\|\nu_{*} /\left(1-|\mu|^{2}\right)^{-1}\right\|_{\infty}: \phi_{\mathbf{T}}^{\prime}(\mu) \nu_{*}=\phi_{\mathbf{T}}^{\prime}(\mu) \nu\right\}
$$

on the tangent bundle $\mathcal{T}(\mathbf{T})=\mathbf{T} \times \mathbf{B}$ of $\mathbf{T}$ (here $\mu \in \operatorname{Belt}(\Delta)_{1}$ and $\nu, \nu_{*} \in L_{\infty}(\mathbb{C})$ ). This structure is locally Lipschitz (cf. [3]).

The smallest dilatation $k(f)=\inf \left\|\mu_{\hat{f}}\right\|_{\infty}$ among quasiconformal extensions of $f \mid \Delta^{*}$ onto $\widehat{\mathbb{C}}$ is called the Teichmüller norm (or dilatation) of $f$.

The space $\mathbf{T}$ as a complex Banach manifold also has invariant metrics (with respect to its biholomorphic automorphisms); the largest and the smallest invariant metrics are the Kobayashi and the Carathéodory metrics, respectively. Namely, the Kobayashi metric $d_{\mathbf{T}}$ on $\mathbf{T}$ is the largest pseudometric $d$ on $\mathbf{T}$ which does not get increased by holomorphic maps $h: \Delta \rightarrow \mathbf{T}$ so that for any two points $\psi_{1}, \psi_{2} \in \mathbf{T}$,

$$
d_{\mathbf{T}}\left(\psi_{1}, \psi_{2}\right) \leq \inf \left\{d_{\Delta}(0, t): h(0)=\psi_{1}, h(t)=\psi_{2}\right\},
$$

where $d_{\Delta}$ is the hyperbolic metric on $\Delta$ with the differential form $\lambda_{\Delta}|d z|$. 
This distance is connected with the Teichmüller norm of $f$ by $k(f)=$ $\tanh d_{\mathbf{T}}\left(\mathbf{0}, S_{f}\right)$.

The Carathéodory distance between $\psi_{1}$ and $\psi_{2}$ in $\mathbf{T}$ is

$$
c_{\mathbf{T}}\left(\psi_{1}, \psi_{2}\right)=\sup d_{\Delta}\left(h\left(\psi_{1}\right), h\left(\psi_{2}\right)\right),
$$

where the supremum is taken over all holomorphic maps $h: \mathbf{T} \rightarrow \Delta$.

The corresponding differential (infinitesimal) forms of these metrics are defined for the points $(\psi, v) \in \mathcal{T}(\mathbf{T})$, respectively, by

$$
\begin{aligned}
\mathcal{K}_{\mathbf{T}}(\psi, v) & =\inf _{h}\{|t|: t \in \mathbb{C}, h \in \operatorname{Hol}(\Delta, \mathbf{T}), h(0)=\psi, d h(0) t=v\} \\
& =\inf _{h}\left\{1 / r: r>0, h \in \operatorname{Hol}\left(\Delta_{r}, \mathbf{T}\right), h(0)=\psi, h^{\prime}(0)=v\right\}, \\
\mathcal{C}_{\mathbf{T}}(\psi, v) & =\sup _{g}\{|d g(\psi) v|: g \in \operatorname{Hol}(\mathbf{T}, \Delta), g(\psi)=0\},
\end{aligned}
$$

where $\operatorname{Hol}(X, Y)$ denotes the collection of holomorphic maps of a complex manifold $X$ into $Y$, and $\Delta_{r}$ is the disk $\{|z|<r\}$. Without normalizing $h(0)=\psi$ and $g(\psi)=0$, one must use instead of $|t|$ and $|d g(\psi) v|$ the hyperbolic lengths $\|t\|_{h^{-1}(\psi)}=|t| /\left(1-\left|h^{-1}(\psi)\right|\right)^{2}$ and $\|d g(\psi) v\|_{g(\psi)}$ of vectors $t$ and $d g(\psi) v$ at the points given by subscripts.

The Royden-Gardiner theorem states that the Kobayashi and Teichmüller metrics are equal on all Teichmüller spaces (cf. $[5-7,24])$. This fundamental fact underlies many applications of Teichmüller space theory. Its strengthened version for the universal Teichmüller space is given by

Proposition 2.1 ([13]). The differential Kobayashi metric $\mathcal{K}_{\mathbf{T}}(\psi, v)$ on the tangent bundle $\mathcal{T}(\mathbf{T})$ of the universal Teichmüller space $\mathbf{T}$ is logarithmically plurisubharmonic in $\psi \in \mathbf{T}$, equals the canonical Finsler structure $F_{\mathbf{T}}(\psi, v)$ on $\mathcal{T}(\mathbf{T})$ generating the Teichmüller metric of $\mathbf{T}$ and has constant holomorphic sectional curvature -4 .

It implies that the Teichmüller distance $\tau_{\mathbf{T}}(\varphi, \psi)$ is logarithmically plurisubharmonic in each of its variables and hence the pluricomplex Green function of the space $\mathbf{T}$ (i.e., the maximal plurisubharmonic function on $\mathbf{T}$ in each of variables $\varphi, \psi)$ equals

$$
g_{\mathbf{T}}(\varphi, \psi)=\log \tanh \tau_{\mathbf{T}}(\varphi, \psi)=\log k(\varphi, \psi),
$$

where $k(\varphi, \psi)$ denotes the extremal dilatation of quasiconformal maps determining the Teichmüller distance between the points $\varphi$ and $\psi$ in $\mathbf{T}$. 


\subsection{Sectional holomorphic curvature}

The generalized Gaussian curvature $\kappa[\lambda]$ of an upper semicontinuous Finsler metric $d s=\lambda(t)|d t|$ with $\lambda(t) \geq 0$ in a domain $\Omega \subset \mathbb{C}$ is defined (at the points $t$, where $\lambda(t) \neq 0$ ) by

$$
\kappa[\lambda](t)=-\frac{\Delta \log \lambda(t)}{\lambda(t)^{2}},
$$

where $\boldsymbol{\Delta}$ is the generalized Laplacian defined by

$$
\Delta \lambda(t)=4 \liminf _{r \rightarrow 0} \frac{1}{r^{2}}\left\{\frac{1}{2 \pi} \int_{0}^{2 \pi} \lambda\left(t+r e^{i \theta}\right) d \theta-\lambda(t)\right\}
$$

(provided that $0 \leq \lambda(t)<\infty)$. It is well known that an upper semicontinuous function $\lambda$ is subharmonic on $\Omega$ if and only if $\Delta u(t) \geq 0$ on this domain; hence at the points $t_{0}$ of local maxima of $\lambda$ with $\lambda\left(t_{0}\right)>-\infty$, we have $\boldsymbol{\Delta} \lambda\left(t_{0}\right) \leq 0$. Note that for $C^{2}$ functions, $\boldsymbol{\Delta}$ coincides with the usual Laplacian $4 \partial^{2} / \partial z \partial \bar{z}$, and its non-negativity immediately follows from the mean value inequality. For arbitrary subharmonic functions, this is obtained by a standard approximation, which also yields the invariance of the generalized Gaussian curvature under complex holomorphic maps (apart from their critical points).

It follows from (2.2) that a (generically nonsmooth) conformal Finsler metric $d s=\lambda(z)|d z|$ with $\lambda(z) \geq 0$ of generalized curvature at most $-K, K>0$, satisfies the inequality

$$
\Delta \log \lambda \geq K \lambda^{2} .
$$

The sectional holomorphic curvature of a Finsler metric on a complex Banach manifold $X$ is defined in a similar way as the supremum of the curvatures (2.2) over appropriate collections of holomorphic maps from the disk into $X$ for a given tangent direction in the image.

The holomorphic curvature of the Kobayashi metric $\mathcal{K}_{X}(x, v)$ of any complete hyperbolic manifold $X$ satisfies $\kappa\left[\mathcal{K}_{X}\right](x, v) \geq-4$ at all points $(x, v)$ of the tangent bundle $\mathcal{T}(X)$ of $X$, and for the Carathéodory metric $\mathcal{C}_{X}$ we have $\kappa\left[\mathcal{C}_{X}\right](x, v) \leq-4$.

For details and general properties of invariant metrics, we refer to $[2,9]$ (see also $[1,13])$.

\subsection{The Grunsky operator}

The complex geometry of the universal Teichmüller space is closely connected with the Grunsky inequalities technique which arose from investigating the univalence problem in [8]. 
Any function $f=z+b_{0}+b_{1} z^{-1}+\cdots \in \Sigma$ determines its Grunsky operator (matrix) $\mathcal{G}_{f}=\left(\alpha_{m n}(f)\right)$, where the Grunsky coefficients $\alpha_{m n}$ are determined by the expansion

$$
\log \frac{f(z)-f(\zeta)}{z-\zeta}=-\sum_{m, n=1}^{\infty} \alpha_{m n} z^{-m} \zeta^{-n}, \quad(z, \zeta) \in\left(\Delta^{*}\right)^{2},
$$

with the principal branch of the logarithmic function, satisfy the inequalities

$$
\left|\sum_{m, n=1}^{\infty} \sqrt{m n} \alpha_{m n} x_{m} x_{n}\right| \leq k .
$$

Here $\mathbf{x}=\left(x_{n}\right)$ runs over the unit sphere $S\left(l^{2}\right)$ of the Hilbert space $l^{2}$ with norm $\|\mathbf{x}\|=\left(\sum_{1}^{\infty}\left|x_{n}\right|^{2}\right)^{1 / 2}$, and $k=k(f) \leq 1$ is the Teichmüller norm of $f$ (cf. $[8,19]$ ). The quantity

$$
\varkappa(f)=\sup \left\{\left|\sum_{m, n=1}^{\infty} \sqrt{m n} \alpha_{m n} x_{m} x_{n}\right|: \mathbf{x}=\left(x_{n}\right) \in S\left(l^{2}\right)\right\} \leq 1
$$

is called the Grunsky norm of $f$; it equals the norm of $\mathcal{G}_{f}$ regarded as a linear operator $l^{2} \rightarrow l^{2}$.

The functions with $\varkappa(f)=k(f)$ play a crucial role in applications of Grunsky inequalities; a point is that the set of $S_{f}$, on which $\varkappa(f)<k(f)$, is open and dense in $\mathbf{T}$. One of the underlying facts in applications is the following result.

Proposition 2.2. The equality $\varkappa(f)=k(f)$ for $f \in \Sigma^{0}$ holds if and only if the function $f$ is the restriction to $\Delta^{*}$ of a quasiconformal self-map $w^{\mu_{0}}$ of $\widehat{\mathbb{C}}$ with Beltrami coefficient $\mu_{0}$ satisfying the condition

$$
\sup \left|\left\langle\mu_{0}, \psi\right\rangle_{\Delta}\right|=\left\|\mu_{0}\right\|_{\infty},
$$

where the supremum is taken over holomorphic functions $\psi \in A_{1}^{2}(\Delta)$ with $\|\varphi\|_{A_{1}(\Delta)}=1$, where

$$
A_{1}^{2}=\left\{\psi \in A_{1}(\Delta): \psi=\omega^{2} \text { with } \omega \text { holomorphic on } \Delta\right\} .
$$

In addition, if an extremal quasiconformal map in the equivalence class of $f$ is of Teichmüller type (in other words, represents in $\mathbf{T}$ a Strebel point), then the restriction of $\mu_{0}$ onto the disk $\Delta$ must be of the form

$$
\mu_{0}(z)=k\left|\psi_{0}(z)\right| / \psi_{0}(z) \quad \text { with } \psi_{0} \in A_{1}^{2} .
$$


The proof of this proposition is given in $[11,15]$. It relies on the fact that the Grunsky coefficients $\alpha_{m n}\left(S_{f}\right)$ generate the holomorphic functions

$$
h_{\mathbf{x}}(\varphi)=\sum_{m, n=1}^{\infty} \sqrt{m n} \alpha_{m n}(\varphi) x_{m} x_{n}
$$

where $\varphi=S_{f}$ and $\mathbf{x}=\left(x_{n}\right)$ are the points of the sphere $S\left(l^{2}\right)$, mapping the universal Teichmüller space $\mathbf{T}$ into the unit disk $\Delta$. The restrictions of these functions to the disk $\left\{\phi_{\mathbf{T}}\left(s \mu_{0}\right)\right\}$ determine the Carathéodory distance between the points $S_{f^{s} \mu_{0}}$ and the origin, which by (2.5) equals the Teichmüller distance.

In a special case, when the curve $f\left(S^{1}\right)$ is analytic, the equality $(2.6)$ was obtained by a different method in [20].

Note that holomorphy of the functions (2.7) is a consequence of the fact that the Grunsky coefficients $\alpha_{m n}$ are polynomials of the initial coefficients $b_{1}, \ldots, b_{m+n-1}$ of $f$ combined with the well-known inequality (cf. [22, p. 61]) : for any $1 \leq p \leq M, 1 \leq q \leq N$,

$$
\left|\sum_{m=p}^{M} \sum_{n=q}^{N} \sqrt{m n} \alpha_{m n} x_{m} x_{n}\right|^{2} \leq \sum_{m=p}^{M}\left|x_{m}\right|^{2} \sum_{n=q}^{N}\left|x_{n}\right|^{2} .
$$

We mention also that both Teichmüller and Grunsky norms are continuous logarithmically plurisubharmonic functions on $\mathbf{T}$ (see $[13,26]$ ) and that, by a theorem of Pommerenke and Zhuravlev, any $f \in \Sigma$ with $\varkappa(f) \leq k<1$ has $k_{1}$-quasiconformal extensions to $\widehat{\mathbb{C}}$ with $k_{1}=k_{1}(k) \geq k$ (see [17, pp. 82-84], [22, 29]).

\subsection{A holomorphic homotopy of univalent functions}

One can define for each $f \in \Sigma$ the complex homotopy

$$
f_{t}(z)=t f\left(\frac{z}{t}\right)=z+b_{0} t+b_{1} t^{2} z^{-1}+b_{2} t^{3} z^{-2}+\cdots: \Delta^{*} \times \Delta \rightarrow \widehat{\mathbb{C}}
$$

so that $f_{0}(z) \equiv z$. This implies $S_{f_{t}}(z)=t^{-2} S_{f}\left(t^{-1} z\right)$, and moreover, this point-wise map determines a holomorphic map

$$
h_{f}(t)=S_{f_{t}}(\cdot): \Delta \rightarrow \mathbf{B}
$$

(see, e.g. [12]). The corresponding homotopy disk

$$
\Delta\left(S_{f}\right)=h_{f}(\Delta) \subset \mathbf{T}
$$

is holomorphic at noncritical points of maps (2.9). These disks foliate the space $\mathbf{T}$.

The dilatations of the homotopy maps are estimated by 
Proposition 2.3 ([12]). (a) Each homotopy map $f_{t}$ of $f \in \Sigma$ admits a $k$-quasiconformal extension to the complex sphere $\widehat{\mathbb{C}}$ with $k \leq|t|^{2}$. The bound $k\left(f_{t}\right) \leq|t|^{2}$ is sharp and occurs only for the maps

$$
f_{b_{0}, b_{1}}(z)=z+b_{0}+b_{1} z^{-1}, \quad\left|b_{1}\right|=1
$$

whose homotopy maps have the affine extensions $\widehat{f}_{b_{0}, b_{1} t^{2}}(z)=z+$ $b_{0} t+b_{1} t^{2} \bar{z}$ onto $\Delta$.

(b) If $f(z)=z+b_{0}+b_{m} z^{-m}+b_{m+1} z^{-(m+1)}+\cdots\left(b_{m} \neq 0\right)$ for some integer $m>1$, then the minimal dilatation of extensions of $f_{t}$ is estimated by $k\left(f_{t}\right) \leq|t|^{m+1}$; this bound also is sharp.

In the second case,

$$
h_{f}(0)=h_{f}^{\prime}(0)=\cdots=h_{f}^{(m)}(0)=\mathbf{0}, h_{f}^{(m+1)}(0) \neq \mathbf{0},
$$

and due to [18],

$$
k\left(f_{t}\right)=\frac{m+1}{2}\left|b_{m}\right||t|^{m+1}+O\left(t^{m+2}\right), \quad t \rightarrow 0 .
$$

Each homotopy function $f_{t}$ has an extremal extension onto $\Delta$ with Beltrami coefficient of the form $t\left|\psi_{t}\right| / \psi_{t}$, where $\psi_{t} \in A_{1}$ and

$$
A_{1}=\left\{\psi \in L_{1}(\Delta), \quad \psi \text { holomorphic on } \Delta\right\}
$$

\section{Proof of Theorem 1.1}

We accomplish the proof in two stages.

Step 1. Underlying lemmas. We shall need the following lemmas.

Lemma 3.1. For every $f \in \Sigma$ with expansion

$f(z)=z+b_{0}+b_{m} z^{-m}+b_{m+1} z^{-(m+1)}+\cdots \quad\left(m \geq 1, b_{m} \neq 0 ;|z|>1\right)$,

there exists a number $r_{f}^{\prime}>0\left(r_{f}^{\prime} \leq 1\right)$ such that

(a) if $m$ is odd, then for each $r \leq r_{f}^{\prime}$ all zeros of the defining differential $\psi_{r}$ in $\Delta$ are of even order;

(b) if $m$ is even, then all $\psi_{r}$ with $r \leq r_{f}$ have only zeros of odd order.

Proof. Investigating the asymptotic behavior of $\varkappa\left(f_{r}\right)$ near $r=0$, one can assume that $[f]$ is a Strebel point, i.e., that $f$ has Teichmüller extension to $\Delta$ with Beltrami coefficient $\mu_{0}=k\left|\psi_{0}\right| / \psi_{0}$ (cf. [7,27]). Otherwise, one 
can replace $f$ by $f_{\rho}$ with $\rho$ close to 1 . Then $f_{\rho \tau}=f_{r}$ for $\tau=r / \rho$. Put $\mu_{0}^{*}=\mu_{0} /\left\|\mu_{0}\right\|_{\infty}$ and

$$
\mu_{r}^{*}(z)= \begin{cases}\frac{\left|\psi_{0}(z / r)\right|}{\psi_{0}(z / r)}, & |z|<r \\ 0, & |z|>r\end{cases}
$$

then for any $\psi \in A_{1}$,

$$
\iint_{\Delta} \mu_{r}^{*}(z) \psi(z) d x d y=r^{2} \iint_{\Delta} \mu_{0}^{*}(z) \psi(r z) d x d y
$$

Note that Beltrami coefficient $k \mu_{r}^{*}$ is extremal in the class of quasiconformal extensions of the stretching $f_{r}(z)=r^{-1} f(z / r)$ across the circle $\{|z|=r\}$.

Consider, for a fixed $r$, the functions

$$
\psi_{r}(z)=r^{-1} \psi(r z)=c_{0}+c_{1} r z+\cdots
$$

generated by $\psi(z)=\sum_{0}^{\infty} c_{n} z^{n} \in A_{1}(\Delta)$. Any such $\psi_{r}$ is holomorphic on the disk $\Delta_{1 / r}=\{|z|<1 / r\}$. The Schwarz lemma, applied to holomorphic maps from $\Delta$ into a ball in $A_{1}$, yields the existence of $r_{0}>0$ depending only on $\|\psi\|_{A_{1}}$ such that $\psi_{r}$ does not vanish on $\Delta \backslash\{0\}$, whenever $r \leq r_{0}$. One derives from Proposition 2.2 and (3.2) that the subspace $A_{1}^{0}$, spanned in $A_{1}(\Delta)$ by these functions, has a nonempty complement in $A_{1}(\Delta)$.

Now observe that the Teichmüller distance between the origin of $\mathbf{T}$ and the equivalence classes $[t \mu]$ for $\mu \in \operatorname{Belt}(\Delta)_{1}$ with $0<\|\mu\|_{\infty} \leq k_{1}<$ 1 and small $t>0$ is given by

$$
\tau_{\mathbf{T}}([0],[t \mu])=t \sup \left|\iint_{\Delta} \mu(z) \psi(z) d x d y\right|+O\left(t^{2}\right),
$$

where the supremum is over all $A_{1}(\Delta)$ for which $\|\psi\|=1$ and the constant in $O\left(t^{2}\right)$ depends only on $k_{1}$ (in fact, this holds for maps of arbitrary Riemann surfaces, cf. [7,10]). We apply (3.3) to the homotopy maps $f_{r}$ with sufficiently small $r$. Let $\mu_{r, 0}$ denote its extremal Beltrami coefficient among quasiconformal extensions of $f_{r}$ across the unit circle $S^{1}$.

By (3.2) the initial coefficient $\mu_{0}$ of $f$ determines a linear bounded functional $l(\psi)$ on $A_{1}^{0}$, which we extend by Hahn-Banach to $A_{1}$ and then to $L_{1}(\Delta)$ getting a Beltrami coefficient $\nu_{r}$ of Teichmüller type. It must coincide with $\mu_{r, 0}$ up to a quantity $O\left(t^{2}\right)$, hence up to an infinitesimally trivial Beltrami coefficient satisfying

$$
\nu \in A_{1}(\Delta)^{\perp}=\left\{\nu_{*} \in \operatorname{Belt}(\Delta)_{1}, \quad\left\langle\nu_{*}, \psi\right\rangle_{\Delta}=0 \text { for all } \psi \in A_{1}\right\} .
$$


As is well known, such a relation is impossible for Teichmüller extremal coefficients; hence, $\nu_{r}=\mu_{r, 0}$.

On the other hand, each $f^{\mu} \in \Sigma^{0}$ with small $\|\mu\|_{\infty}$ is represented by the well-known variational formula

$$
f^{\mu}(\zeta)=\zeta+b_{0}-\frac{1}{\pi} \iint_{\Delta} \frac{\mu(z)}{z-\zeta} d x d y+O\left(\|\mu\|_{\infty}^{2}\right)=\zeta+b_{0}+\sum_{1}^{\infty} b_{n} \zeta^{-n}
$$

with

$$
b_{n}=\frac{1}{\pi} \iint_{\Delta} \mu(z) z^{n-1} d x d y+O\left(\|\mu\|_{\infty}^{2}\right), \quad n=1,2, \ldots
$$

which yields

$$
\alpha_{m n}(\varphi)=-\frac{1}{\pi} \iint_{\Delta} \mu(z) z^{m+n-2} d x d y+O\left(\|\mu\|_{\infty}^{2}\right), \quad\|\mu\|_{\infty} \rightarrow 0 .
$$

Comparison with (3.3), Proposition 2.2 and definition of the Grunsky norm immediately implies $\varkappa\left(f_{r}\right)<k\left(f_{r}\right)$ if $m$ in (3.1) is even, and

$$
\varkappa\left(f_{r}\right)=k\left(f_{r}\right)=\left|\mu_{r, 0}\right| \quad\left(\text { for } r \leq r_{1}(f)\right)
$$

if $m$ is odd. These relations are equivalent to assertions $(a),(b)$. Lemma 3.1 follows.

Remark 3.1. It follows from Proposition 2.2 that there exist $f(z)=$ $z+b_{0}+b_{1} z^{-1}+\cdots \in \Sigma^{0}$ with $b_{1} \neq 0$ and $\varkappa(f)<k(f)$. Lemma 3.1 shows that the homotopy maps $f_{r}$ with sufficiently small $r<r_{f}$ inherit the relation between Teichmüller and Grunsky norms of the tangent maps $f_{m, r}(z)=f_{0, b_{1} r^{2}}\left(z^{m}\right)^{1 / m}$ at $r=0$.

The assertion (a) of Lemma 3.1 for $f \in \Sigma$ with $b_{1} \neq 0$ was established by R. Kühnau (by a different method). In [18, Section 6] he posed some related conjectures. Lemma 3.1 generalizes his result and partially answers some of Kühnau's questions.

We apply this lemma to establish the equality of hyperbolic distances for the points of homotopy disks $\Delta\left(f_{t}\right)$ in $\mathbf{T}$ near the origin.

Lemma 3.2. For every $f \in \Sigma$ of the form (3.1) with $m \geq 1$, there exists $r_{f}>0$ such that for all $r \leq r_{f}$, we have the equalities

$$
\begin{aligned}
c_{\mathbf{T}}\left(\mathbf{0}, S_{f_{r}}\right)=d_{\mathbf{T}}\left(\mathbf{0}, S_{f_{r}}\right) & =\tau_{\mathbf{T}}\left(\mathbf{0}, S_{f_{r}}\right) \\
& =\tanh ^{-1}\left(\frac{m+1}{2}\left|b_{m}\right| r^{m+1}\right)+O\left(r^{m+2}\right) .
\end{aligned}
$$

The infinitesimal forms of these metrics also coincide for small $r$. 
Proof. First assume that $m$ in expansion (3.1) is odd. Then by Lemma 3.1 there exists $r_{f}>0$ such that for any $r \leq r_{f}$ the extremal Beltrami coefficient of the homotopy map $f_{r}$ is of the form

$$
\mu_{r}=k_{r}\left|\psi_{r}\right| / \psi_{r} \quad \text { with } \quad \psi_{r} \in A_{1}^{2},
$$

i.e., $\psi_{r}=\omega_{r}^{2}$ with holomorphic $\omega_{r}$ in $\Delta$. Such a quadratic differential is represented in the form

$$
\psi_{r}(z)=\frac{1}{\pi} \sum_{m+n=2}^{\infty} \sqrt{m n} x_{m}^{r} x_{n}^{r} z^{m+n-2}
$$

with $\mathbf{x}^{r}=\left(x_{n}^{r}\right) \in l^{2}$ and $\|\psi\|_{A_{1}}=\left\|\mathbf{x}^{0}\right\|_{l^{2}}$ (cf. [20]). Pick $c>0$ so that $\left\|c \psi_{r}\right\|_{A_{1}}=1$, and consider the holomorphic map

$$
h_{c \mathbf{x}^{0}}(\varphi)=\sum_{m, n=1}^{\infty} \sqrt{m n} \alpha_{m n}(\varphi) x_{m}^{0} x_{n}^{0}: \mathbf{T} \rightarrow \Delta
$$

whose lifting into the ball $\operatorname{Belt}(\Delta)_{1}$ is $\widehat{h}_{c \mathbf{x}^{0}}(\mu)=h_{c \mathbf{x}^{0}} \circ \phi_{\mathbf{T}}$. Using (3.4), one computes that the differential of $\widehat{h}_{c \mathbf{x}^{0}}$ at the origin $\mu=\mathbf{0}$ equals

$$
d \widehat{h}_{c \mathbf{x}^{0}}(\mathbf{0}) \nu=\frac{1}{\pi} \iint_{\Delta} \nu(z) \sum_{m, n=1}^{\infty} \sqrt{m n} x_{m}^{0} x_{n}^{0} z^{m+n-2} d x d y .
$$

In particular, for $\nu=t \mu_{r}$,

$$
d \widehat{h}_{c \mathbf{x}^{0}}(\mathbf{0})\left[t\left|\psi_{r}\right| / \psi_{r}\right]=c t \iint_{\Delta}\left|\psi_{r}(z)\right| d x d y=t,
$$

hence the restriction of the map (3.7) to the disk $\Delta\left(\psi_{r}\right)=\left\{\phi_{\mathbf{T}}\left(\left\{t \mu_{r}\right\}\right)\right\} \subset$ $\mathbf{T}$ is a hyperbolic isometry of the unit disk, and the Carathéodory and Teichmüller metrics of $\mathbf{T}$ are equal on $\Delta\left(\psi_{r}\right)$. Since $c_{\mathbf{T}}(\cdot, \cdot) \leq d_{\mathbf{T}}(\cdot, \cdot) \leq$ $\tau_{\mathbf{T}}(\cdot, \cdot)$, the equalities (3.6) follow.

If $m$ in (3.1) is even, we consider the map

$$
f_{2}(z)=f\left(z^{2}\right)^{1 / 2}=z+\frac{b_{0}}{2} \frac{1}{z}+\frac{b_{m}}{2} \frac{1}{z^{2 m-1}}+\cdots
$$

It is well-defined since $f(0)=0$ and provides an odd function, symmetric with respect to the origin. Denote the coefficients of $f_{2}$ by $b_{j}^{(2)}$ and let $\alpha_{m n}^{(2)}(f)=\alpha_{m n}\left(f_{2}\right)$.

Squaring $f(z) \mapsto f\left(z^{2}\right)^{1 / 2}$ transforms the defining quadratic differentials $\psi_{r}=\psi_{r}(z) d z^{2}$ of $f_{r}$ into $\psi_{r}^{*}=\psi_{r}\left(z^{2}\right) 4 z^{2} d z^{2}$ with zero of even order 
at the origin. Thus one can apply to $f_{2}$ the previous arguments, using instead of (2.10) the functions

$$
h_{2, \mathbf{x}}(\varphi)=\sum_{m, n=1}^{\infty} \sqrt{m n} \alpha_{m n}^{(2)}(\varphi) x_{m} x_{n}
$$

The maps $f$ are completely normalized, thus each coefficient $b_{j}, j \geq 0$, is holomorphic on $\mathbf{T}$. It is represented as a polynomial of a finite number of initial coefficients $b_{0}, b_{1}, \ldots, b_{s}$ of the original function $f$ (and free terms $b_{0}$ are uniquely determined from the condition $f(0)=0$ ). Thus the maps (3.8) also depend holomorphically from $\mu_{f}$ and $S_{f}$, and since squaring transform preserves quasiconformal dilatation of $f_{r}$, the equalities (3.6) follow.

The proof for the infinitesimal metrics follows the same line, completing the proof of the lemma.

Step 2. Equality of invariant metrics on the homotopy disks. First assume that $S_{f}$ is a Strebel point of the space $\mathbf{T}$, equivalently, $f \in \Sigma^{0}$ admits quasiconformal extension whose Beltrami coefficient in $\Delta$ is defined by a holomorphic quadratic differential $\psi \in A_{1}$. We compare the infinitesimal Carathéodory and Kobayashi metrics on the homotopy disk $\Delta\left(S_{f}\right)$, using Minda's maximum principle.

Lemma 3.3 ([21]). If a function $u: \Omega \rightarrow[-\infty,+\infty)$ is upper semicontinuous in a domain $\Omega \subset \mathbb{C}$ and its generalized Laplacian satisfies the inequality $\boldsymbol{\Delta} u(z) \geq K u(z)$ with some positive constant $K$ at any point $z \in \Omega$, where $u(z)>-\infty$, and if

$$
\limsup _{z \rightarrow \zeta} u(z) \leq 0 \quad \text { for all } \zeta \in \partial \Omega,
$$

then either $u(z)<0$ for all $z \in \Omega$ or else $u(z)=0$ for all $z \in \Omega$.

The restrictions of the infinitesimal metrics Carathéodory and Kobayashi metrics to the disk $\Delta\left(S_{f}\right)$ determine on $\Delta$ the metrics

$$
\begin{aligned}
& \lambda_{\mathcal{K}}(t)=\mathcal{K}_{\mathbf{T}}\left(h_{f}(t), h_{f}^{\prime}(t)\right)=\frac{|t|}{\left(1-\left|h_{f}^{-1}\left(S_{f_{t}}\right)\right|\right)^{2}}, \\
& \lambda_{\mathcal{C}}(t)=\mathcal{C}_{\mathbf{T}}\left(h_{f}(t), h_{f}^{\prime}(t)\right)=\sup _{g}\left\{\frac{\left|g \circ h_{f}(t)\right|}{1-\left|g \circ h_{f}(t)\right|^{2}}: g \in \operatorname{Hol}(\mathbf{T}, \Delta)\right\}
\end{aligned}
$$

having at noncritical points of the map $h_{f}$ the generalized Gaussian curvatures -4 and at most -4 , respectively. By Lemma 3.2, for all $|t| \leq r_{f}$, 
we have the equality $c_{\mathbf{T}}\left(\mathbf{0}, S_{f_{t}}\right)=d_{\mathbf{T}}\left(\mathbf{0}, S_{f_{t}}\right)$, which implies, by Schwarz's lemma,

$$
\lambda_{\mathcal{C}}(t)=\lambda_{\mathcal{K}}(t), \quad \text { for }|t|<\min \left\{r_{f}, 1\right\},
$$

while $\lambda_{\mathcal{C}}(t) \leq \lambda_{\mathcal{K}}(t)$ for $r_{f}<|t|<1$. We only need to consider the case $r_{f}<1$.

Apart from the critical points of the map $h_{f}$, the metric $\lambda_{\mathcal{C}}(t)$ is subharmonic, hence upper semicontinuous, and $\lambda_{\mathcal{K}}(t)$ is locally Lipschitz continuous and subharmonic. Thus if there are the points $t \in \Delta$, where $\left.\lambda_{\mathcal{C}}(t)<\lambda_{\mathcal{K}}(t)\right\}$, their set must be open. Pick a connected component $\Omega_{0}$ of this set and a boundary point $t_{0}$ of $\Omega_{0}$, and put

$$
M=\left\{\sup \lambda_{\mathcal{K}}(t): t \in U_{0}\right\}
$$

for $t$ running over a sufficiently small neighborhood $U_{0}$ of $t_{0}$. For all such $t, \quad \lambda_{\mathcal{K}}(t)+\lambda_{\mathcal{C}}(t) \leq 2 M ;$ thus, letting

$$
u=\log \left(\lambda_{\mathcal{C}} / \lambda_{\mathcal{K}}\right)
$$

we get

$$
\begin{aligned}
\Delta u(t)=\Delta \log \lambda_{\mathcal{C}}(t) & -\Delta \log \lambda_{\mathcal{K}}(t) \\
= & 4\left(\lambda_{\mathcal{C}}(t)^{2}-\lambda_{\mathcal{K}}(t)^{2}\right) \geq 8 M\left(\lambda_{\mathcal{C}}(t)-\lambda_{\mathcal{K}}(t)\right)
\end{aligned}
$$

Since

$$
M \log (a / b) \geq a-b \quad \text { for } 0<b \leq a<M
$$

(with equality only for $a=b)$, (3.10) yields

$$
M \log \frac{\lambda_{\mathcal{C}}(t)}{\lambda_{\mathcal{K}}(t)} \geq \lambda_{\mathcal{C}}(t)-\lambda_{\mathcal{K}}(t),
$$

and hence

$$
\Delta u(t) \geq 4 M^{2} u(t) .
$$

The properties of $\lambda_{\mathcal{C}}$ and $\lambda_{\mathcal{K}}$ yield that $u$ is upper semicontinuous.

We can apply Lemma 3.3 which provides, in view of (3.9), that both metrics $\lambda_{\mathcal{C}}$ and $\lambda_{\mathcal{K}}$ must be equal on $U_{0}$, hence in some domain containing the initial disk $\left\{|t|<r_{f}\right\}$. Continuing in a similar way, one obtains the equality of these metrics at all noncritical points of the map $h_{f}$. This yields that the original metrics $\mathcal{C}_{\mathbf{T}}$ and $\mathcal{K}_{\mathbf{T}}$ coincide at all nonsingular points of the disk $\Delta\left(S_{f}\right)$.

Now consider the Teichmüller disks containing the origin $\psi=\mathbf{0}$ and touching $\Delta\left(S_{f}\right)$ at such points, and compose on these disks the corresponding holomorphic maps $\Delta \rightarrow \mathbf{T}$ and $\mathbf{T} \rightarrow \Delta$ defining the distances 
$d_{\mathbf{T}}$ and $c_{\mathbf{T}}$. The Schwarz lemma implies, in view of the established equality of the infinitesimal metrics, that $c_{\mathbf{T}}=d_{\mathbf{T}}$ on any such disk. By continuity of metrics, this equality extends to all points of the homotopy disk $\Delta\left(S_{f}\right)$.

Now observe that $d_{\mathbf{T}}=\tau_{\mathbf{T}}$ (and hence also $c_{\mathbf{T}}$ ) is circularly symmetric on $\Delta\left(S_{f}\right)$ (i.e., $d_{\mathbf{T}}\left(\mathbf{0}, S_{f_{t}}\right)=d_{\mathbf{T}}\left(\mathbf{0}, S_{f_{\mid} t \mid}\right)$ for all $\left.|t| \leq 1\right)$. Since any circularly symmetric subharmonic function $u(t)$ on $\Delta$ is continuous in $|t| \in[0,1]$, one gets

$$
c_{\mathbf{T}}\left(\mathbf{0}, S_{f}\right)=d_{\mathbf{T}}\left(\mathbf{0}, S_{f}\right) .
$$

To establish the equality of infinitesimal metrics, consider the Teichmüller geodesic disk $\left\{\phi_{\mathbf{T}}\left(s \mu_{0}\right):|s|<1\right\}$ joining the point $S_{f}$ with the origin of $\mathbf{T}$. Let $S_{f}$ correspond to $s=s_{0}$. Pick in $\operatorname{Hol}(\mathbf{T}, \Delta)$ a sequence of maps $g_{n}$ for which

$$
\lim _{n \rightarrow \infty} d_{\Delta}\left(\mathbf{0}, g_{n}\left(S_{f}\right)\right)=c_{\mathbf{T}}\left(\mathbf{0}, S_{f}\right) .
$$

Then Schwarz's lemma, applied to the functions

$$
\widehat{g}_{n}(s)=g_{n}\left(S_{f^{s \mu_{0}}}\right): \Delta \rightarrow \Delta, \quad n=1,2, \ldots,
$$

implies, in view of (3.11),

$$
\lim _{n \rightarrow \infty} d g_{n}\left(S_{f^{s_{0}} \mu_{0}}\right) v=1, \quad v=\phi_{\mathbf{T}}\left(\mu_{0}\right),
$$

which, in turn, yields the equality

$$
\mathcal{C}_{\mathbf{T}}\left(S_{f}, v\right)=\mathcal{K}_{\mathbf{T}}\left(S_{f}, v\right)
$$

for all tangent vectors $v$ at $S_{f}$. This completes the proof of the theorem for Strebel's points.

Using the density of such points in the space $\mathbf{T}$ and the continuity of metrics, one extends the equality (3.11) to all points of $\mathbf{T}$. Then the case of the infinitesimal metrics is investigated in the same way as for Strebel's points using one of the extremal Beltrami coefficients $\mu_{0}$ for $f$ (now not unique) and applying the above arguments to the disk $\left\{\phi_{\mathbf{T}}\left(s \mu_{0}\right)\right\}$.

It remains to establish the coincidence of the global distances between arbitrary points $\varphi_{1}, \varphi_{2}$ in $\mathbf{T}$. One can use for this a standard procedure involving the so-called right translations of $\mathbf{T}_{1}$, generated by the right shifts on the ball $\operatorname{Belt}(\Delta)_{1}$.

Let us take a Beltrami coefficient $\nu \in \operatorname{Belt}(\Delta)_{1}$ with $\phi_{\mathbf{T}}(\nu)=\varphi$. Then $w^{\nu}\left(S^{1}\right)$ is a quasicircle with the interior domain $D_{\nu}=w^{\nu}(\Delta)$. 
Having this domain mapped conformally onto $\Delta$ by the corresponding function $w$, one obtains (for fixed $\nu$ ) a biholomorphic isomorphism

$$
\mu \mapsto \sigma(\mu)=\frac{\mu-\nu}{1-\bar{\nu} \mu} \frac{w^{\nu} \circ w^{-1}}{\overline{w^{\nu} \circ w^{-1}}}
$$

of the ball $\operatorname{Belt}(\Delta)_{1}$. This isomorphism is compatible with the canonical projection $\phi_{\mathbf{T}}$ and thus descends to a holomorphic bijection $\widehat{\sigma}$ of $\mathbf{T}$ defined from the commutative diagram

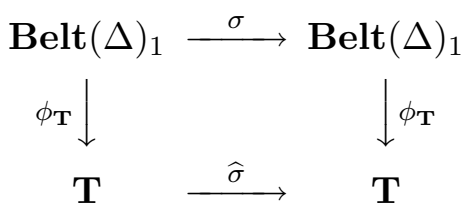

It implies also the Teichmüller isometry

$$
\tau_{\mathbf{T}}\left(\phi_{\mathbf{T}}(\nu), \phi_{\mathbf{T}}(\mu)\right)=\tau_{\mathbf{T}}\left(\phi_{\mathbf{T}}(\sigma(\nu)), \mathbf{0}\right)
$$

and the similar equalities for the Carathéodory and Kobayashi metrics. This completes the proof of the theorem.

\section{Extremal problems for univalent functions}

\subsection{Complex geodesics}

By Schwarz's lemma, any holomorphic map $j$ of a complex Banach manifold $X$ into the disk $\Delta$ is estimated by

$$
c_{X}(x, y) \leq d_{X}(x, y) \leq d_{\Delta}(j(x), j(y)), \quad x, y \in X,
$$

where $c_{X}$ and $D_{X}$ denote the Carathéodory and Kobayashi metrics of $X$, respectively.

A holomorphic map $h: \Delta \rightarrow X$ is called a complex geodesic if for any two distinct points $t_{1}, t_{2} \in \Delta$,

$$
d_{\Delta}\left(t_{1}, t_{2}\right)=c_{X}\left(h\left(t_{1}\right), h\left(t_{2}\right)\right)=d_{X}\left(h\left(t_{1}\right), h\left(t_{2}\right)\right) .
$$

Then $h(\Delta)$ is a holomorphically embedded disk, geodesic for both metrics $c_{X}$ and $d_{X}$ (cf. [28]).

\subsection{A general theorem}

Now let $F(f)$ be a holomorphic (continuous and Gateaux $\mathbb{C}$-differentiable) functional on the class $\Sigma$, i.e., we have for any $f \in \Sigma$ and small $t \in \mathbb{C}$ the equality

$$
F(f+t h)=F(f)+t F_{f}^{\prime}(h)+O\left(t^{2}\right), \quad t \rightarrow 0,
$$


in the topology of uniform convergence on compact sets in $\Delta^{*}$. Here $F_{f}^{\prime}(h)$ is a $\mathbb{C}$-linear functional. The restriction of $F$ to $\Sigma^{0}$ is lifted to the ball $\operatorname{Belt}(\Delta)_{1}$ by $\widehat{F}(\mu)=F\left(f^{\mu}\right)$, and we assume that this lifting is holomorphic on $\operatorname{Belt}(\Delta)_{1}$. Moreover, assume that this functional is holomorphic on the Schwarzians $S_{f^{\mu}}$. Then the functional $F_{f}^{\prime}(h)$ in (4.1) is a strong (Fréchet) derivative of $F$ on $\mathbf{T}$.

We already used in the proof of Lemma 3.1 a variational formula for $f \in \Sigma^{0}$. More generally,

$$
\omega=H^{\mu}(w)=w+\frac{1}{\pi} \iint_{f\left(\Delta^{*}\right)} \mu(\zeta) g(w, \zeta) d \xi d \eta+O\left(\|\mu\|_{\infty}^{2}\right) ;
$$

where the kernel $g$ of variation is of the form

$$
g(w, \zeta)=\frac{1}{w-\zeta}+g_{1}(\zeta)
$$

where the additional term $g_{1}(\zeta)$ depends on the additional normalization of $f$. For example, if $f(1)=1, f(0)=0, b_{0}(f)=0$, then $g_{1}(\zeta)$ equals, respectively, to $1 / \zeta, 1 /(\zeta-1), 0$. Under any of such normalizations, the ratio $O\left(\|\mu\|_{\infty}^{2}\right) /\|\mu\|_{\infty}^{2}$ remains uniformly bounded on $\widehat{\mathbb{C}}$ as $\|\mu\| \rightarrow 0$. We shall assume that such an additional normalization of $f \in \Sigma^{0}$ is chosen.

As is well known, any such $F$ is represented by a complex Borel measure on $\mathbb{C}$, which allows to extend this functional to all holomorphic functions on $\Delta^{*}$ (cf. [25]). In particular, the value $F_{I}(g(I, z))$ of $F$ on the identity map $I(z)=z$ is well defined.

Let us consider the case when the derivative

$$
\psi_{0}(z)=F_{I}^{\prime}(g(I, z))
$$

is a meromorphic functions on $\mathbb{C}$, which is holomorphic and integrable on the unit disk $\Delta$. This rather natural assumption holds, for example, for the general distortion functionals $F$ of the form

$$
F(f)=F\left(f^{\prime}\left(z_{1}\right), \ldots, f^{\left(m_{1}\right)}\left(z_{1}\right) ; \ldots ; f^{\prime}\left(z_{p}\right), \ldots, f^{\left(m_{p}\right)}\left(z_{1}\right)\right),
$$

where $z_{1}, \ldots, z_{p}$ are the distinct fixed points in $\overline{\Delta^{*}}$ with assigned orders $m_{1}, \ldots, m_{p}$, respectively. In this case, the function (4.2) is rational.

The following general theorem immediately solves the maximization problem for any functional $F$ of the above form on the classes

$$
\Sigma_{k}=\left\{f^{\mu} \in \Sigma^{0}:\|\mu\|_{\infty} \leq k\right\} \quad(k<1)
$$

under an appropriate restriction to the dilatation $k$. 
Theorem 4.1. Suppose that the range domain of the functional $F$ on $\Sigma^{0}$ has more than two boundary points and its derivative $F_{I}^{\prime}(g(I, \cdot))=\psi_{0}$ determines in $\mathbf{T}$ a $c_{\mathbf{T}}$-geodesic disk

$$
\Delta\left(\psi_{0}\right)=\left\{\phi_{\mathbf{T}}\left(\left|\psi_{0}\right| / \psi_{0}\right)\right\}
$$

Then there exists a number $k_{0}(F)>0$ such that for all $k \leq k_{0}(F)$, we have the sharp bound

$$
\max _{k(f) \leq k}|F(f)-F(I)| \leq \max _{|t|=k}\left|F\left(f^{t\left|\psi_{0}\right| / \psi_{0}}\right)-F(I)\right|
$$

in other words, the values of $F$ on the ball

$$
\operatorname{Belt}(\Delta)_{k}=\left\{\mu \in \operatorname{Belt}(\Delta)_{1}:\|\mu\|_{\infty} \leq k\right\}
$$

are located in the closed disk $\Delta\left(F(I), M_{k}\right)$ centered at the point $F(I)$ and with radius

$$
M_{k}(F)=\max _{|t|=k}\left|F\left(f^{t\left|\psi_{0}\right| / \psi_{0}}\right)-F(I)\right|
$$

The equality occurs only for $\mu=t\left|\psi_{0}\right| / \psi_{0}$ with $|t|=k$.

Conversely, if the inequality (4.3) holds for $F$ for $0<k \leq k_{0}(F)$, then up to a constant multiple $a>0$,

$$
F\left(f^{\mu}\right)=\widetilde{G}\left(S_{f^{\mu}}\right)+O\left(\|\mu\|_{\infty}^{2}\right) \quad \text { as }\|\mu\|_{\infty} \rightarrow 0
$$

where $\widetilde{G}$ is holomorphic on $\mathbf{T}$ and its renormalization

$$
\widetilde{G}(\varphi)=\widetilde{G}(\varphi) / \sup _{\psi \in \mathbf{T}}|\widetilde{G}(\varphi)|
$$

is the defining map for the disk $\Delta\left(\psi_{0}\right)$ as $c_{\mathbf{T}}$-geodesic.

This theorem implies many sharp distortion estimates for univalent functions with quasiconformal extension. It shows that assumption of the theorem about the disk (4.3) to be $c_{\mathbf{T}}$-geodesic is in fact sufficient and necessary for getting the explicit bound (4.4).

The known results in this direction are of rather specific forms and give only the sufficient conditions ensuring the bound (4.4). For example, it was established in [16] for functionals $F$ whose derivatives $F_{I}^{\prime}(g(I, \cdot))$ have in the unit disk only zeros of even order, using a special projector of norm 1. The same idea underlies the proof of sufficiency in Theorem 4.1.

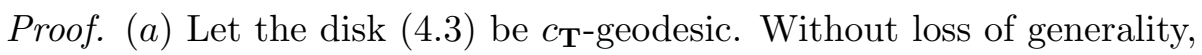
one can assume that $f^{\mu} \in \Sigma^{0}$ are normalized by $f^{\mu}(1)=1$, hence for small $\|\mu\|$, 


$$
\begin{aligned}
f^{\mu}(z)=z & -\frac{1}{\pi} \iint_{\Delta} \mu(\zeta)\left(\frac{1}{\zeta-z}-\frac{1}{\zeta-1}\right) d \xi d \eta+O\left(\|\mu\|_{\infty}^{2}\right) \\
= & z-\frac{z-1}{\pi} \iint_{\Delta} \frac{\mu(\zeta) d \xi d \eta}{(\zeta-1)(\zeta-z)}+O\left(\|\mu\|_{\infty}^{2}\right) \quad \text { as }\|\mu\|_{\infty} \rightarrow 0
\end{aligned}
$$

and $F(I)=0$. Let $f_{0}$ be any function in $\Sigma_{k}$ maximizing $|F|$ over $\Sigma_{k}$ (the existence of such $f_{0}$ follows from compactness). We may assume that its Beltrami coefficient $\mu_{f_{0}}$ is extremal in its class, i.e.,

$$
\left\|\mu_{f_{0}}\right\|_{\infty}=\inf \left\{\|\mu\|_{\infty} \leq k: f^{\mu}\left|\Delta^{*}=f_{0}\right| \Delta^{*}\right\} .
$$

Suppose that

$$
\mu_{f_{0}} \neq t \mu_{0} \quad \text { for some } t \text { with }|t|=k,
$$

where

$$
\mu_{0}=\left|\psi_{0}\right| / \psi_{0} .
$$

We show that for small $k$ this leads to a contradiction.

The proof relies on the following important property of extremal maps. Let

$$
\omega_{p}(z)=z^{p}-1-\psi_{0}(z) \quad(\zeta \in \Delta), \quad p=1,2, \ldots,
$$

and

$$
\langle\mu, \psi\rangle_{\Delta}=\iint_{\Delta} \mu(z) \psi(z) d x d y, \quad \text { for } \mu \in L_{\infty}(\Delta), \quad \psi \in L_{1}(\Delta) .
$$

The following lemma from [16] provides a key property of extremal maps.

Lemma 4.1. For sufficiently small $k \leq k_{0}(F)$, the extremal Beltrami coefficient $\mu_{f_{0}}$ is orthogonal to all functions (4.8), i.e.,

$$
\left\langle\mu_{f_{0}}, \omega_{p}\right\rangle_{\Delta}=0 .
$$

Consider now the holomorphic map

$$
\Lambda(\mu)=g \circ \phi_{\mathbf{T}}(\mu): \mu \in \operatorname{Belt}(\Delta)_{1},
$$

where $g: \mathbf{T} \rightarrow \Delta$ determines the hyperbolic isometry between the disks (4.3) and $\Delta$. The differential of $\Lambda$ at $\mu=\mathbf{0}$ is a linear operator $P$ : $L_{\infty}(\Delta) \rightarrow L_{\infty}(\Delta)$ of norm 1 which is represented in the form

$$
P(\mu)=\beta\left\langle\mu, \psi_{0}\right\rangle \mu_{0} .
$$


Let $P\left(\mu_{f_{0}}\right)=\alpha(k) \mu_{0}$. Since, by assumption, $f_{0}$ is not equivalent to $f^{t_{0} \mu_{0}}$ with $\left|t_{0}\right|=k$, we have

$$
\left\{\Lambda\left(\frac{t}{k} \mu_{f_{0}}\right):|t|<1\right\} \varsubsetneqq\{|t|<1\} .
$$

Thus, by Schwarz's lemma,

$$
|a(k)|<k \text {. }
$$

Now consider the function

$$
\nu_{0}=\mu_{f_{0}}-\alpha(k) \mu_{0}
$$

which is not equivalent to zero, due to our assumption (4.7). We show that $\nu_{0}$ annihilates integrable holomorphic functions on $\Delta$. We use the following lemma from [4] concerning the projections of norm 1 in Banach spaces.

Let $V$ be a complex Banach space with norm $\|\cdot\|$ differentiable on $V \backslash\{0\}$, and suppose that

$$
A(v, w)=\lim _{t \rightarrow 0} \frac{\|v+t w\|-\|v\|}{t} \text { for all } v \in V \backslash\{0\}, w \in V ;
$$

for every fixed $v \neq 0$ it is a bounded linear functional on $V$.

Lemma 4.2. Let $W$ be a non-trivial closed (complex) subspace of $V$, and let $W^{\prime}$ be the closed subspace

$$
W^{\prime}=\{w \in V: A(v, w)=0 \quad \text { for all } v \in W \backslash\{0\} .
$$

There is a projection $P$ of norm 1 from $V$ onto $W$ if and only if $W^{\prime}$ is a complementary subspace to $W$, that is $W \oplus W^{\prime}=V$. Further, if $P$ exists, it is unique and its kernel is $W^{\prime}$.

We have

$$
\left\langle\nu_{0}, \psi\right\rangle_{\Delta}=0
$$

for all $\psi$ from the subspace $W^{\prime}=\left\langle\omega_{p}\right\rangle$ of $A_{1}(\Delta)$ spanned by functions (4.8), since $\left\langle\mu_{f_{0}}, \psi\right\rangle_{\Delta}=0$ by Lemma 4.1 and $\left\langle\mu_{0}, \psi\right\rangle_{\Delta}=0$ by Lemma 4.3 applied to the one-dimensional subspace $W=\left\{\lambda \psi_{0}: \lambda \in \mathbb{C}\right\}$. To establish that

$$
\left\langle\nu_{0}, \psi_{0}\right\rangle_{\Delta}=0,
$$

consider the conjugate operator

$$
P^{*}(\psi)=\left\langle\mu_{0}, \psi\right\rangle_{\Delta} \psi_{0}
$$


which maps $L_{1}(\Delta)$ into $L_{1}(\Delta)$ and fixes the subspace $W$. The definition of $\nu_{0}$ implies $P\left(\nu_{0}\right)=0$, thus

$$
\left\langle\nu_{0}, \psi_{0}\right\rangle_{\Delta}=\lambda\left\langle\nu_{0}, P^{*} \nu_{0}\right\rangle_{\Delta}=\left\langle P \nu_{0}, \psi_{0}\right\rangle_{\Delta}=0 .
$$

Since the functions $\psi_{0}, \omega_{p}, p=1,2, \ldots$, form a complete set in the space $A_{1}(\Delta)$, we have proved that $\nu_{0}$ is orthogonal to all $\psi \in A_{1}(\Delta)$, i.e., belongs to the set

$$
A_{1}(\Delta)^{\perp}=\left\{\mu \in L_{\infty}(\Delta):\langle\mu, \psi\rangle_{\Delta}=0 \text { for all } \psi \in A_{1}(\Delta)\right\} .
$$

Now we use the well-known properties of extremal quasiconformal maps (see e.g., $[7,10,23])$. First of all, since $\mu_{f_{0}}$ is extremal for $f_{0}$,

$$
\left\|\mu_{f_{0}}\right\|_{\infty}=\inf \left\{\left|\left\langle\mu_{f_{0}}, \psi\right\rangle_{\Delta}\right|: \varphi \in A_{1}(\Delta),\|\psi\|=1\right\} ;
$$

moreover, by the Hamilton-Krushkal-Reich-Strebel theorem such an equality is necessary and sufficient for $\mu \in \operatorname{Belt}(\Delta)_{1}$ to be extremal for $f^{\mu}$. Hence, for any $\nu \in A_{1}(\Delta)^{\perp}$,

$$
\left\|\mu_{f_{0}}\right\|_{\infty}=\inf \left\{\mid\left\langle\mu_{f_{0}}+\nu, \psi\right\rangle_{\Delta}: \psi \in A_{1}(\Delta),\|\psi\|=1\right\} \leq\left\|\mu_{f_{0}}+\nu\right\|_{\infty} .
$$

Thus we have

Lemma 4.3. If $f_{0}$ is extremal.

$$
\left\|\mu_{f_{0}}\right\|_{\infty}=k \leq\left\|\mu_{f_{0}}-\nu_{0}\right\|_{\infty} .
$$

We may now complete the proof of the sufficiency part. By (4.10),

$$
k \leq\left\|\mu_{f_{0}}-\nu_{0}\right\|_{\infty}=\left\|\alpha(k) \mu_{0}\right\|_{\infty}=\alpha(k),
$$

which contradicts (4.9). Hence $f_{0}$ is equivalent to $f^{t \mu_{0}}$ and we can take $\mu_{f_{0}}=t \mu_{0}$ for some $|t|=k$.

(b) Let again $F(I)=0$. We lift the original functional $F$ to

$$
G(\mu)=\pi^{-1} \circ \widehat{F}(\mu): \operatorname{Belt}(\Delta)_{1} \rightarrow \Delta,
$$

where $\pi$ is a holomorphic universal covering of the domain $V(F)=$ $F\left(\Sigma^{0}\right)$ by a disk $\Delta_{a}=\{|z|<a\}$ with $\pi(0)=0, \pi^{\prime}(0)=1$ (the lifting is single valued, since the ball $\operatorname{Belt}(\Delta)_{1}$ is simply connected). The normalization of $\pi$ ensures that for sufficiently small $|\zeta|$,

$$
\pi(\zeta)=\zeta+O\left(\zeta^{2}\right)
$$

(with uniform estimate of the remainder for $|\zeta|<\left|\zeta_{0}\right|$ ), which implies the asymptotic equality (4.6). The covering functional $G$ is holomorphic also 
in the Schwarzians $S_{f^{\mu}}$, which generates a holomorphic map $\widetilde{G}: \mathbf{T} \rightarrow \Delta$ so that $G=\widetilde{G} \circ \phi_{\mathbf{T}}$. The above arguments estimate $G$ for small $\|\mu\|$ similarly to (4.4), but now the radius (4.5) is given by

$$
M_{k}(G)=k \quad \text { for } 0<k<k_{1}(g) .
$$

Restricting $\widetilde{G}$ to the extremal disk (4.3) and applying to this restriction Schwarz's lemma, one derives that on this disk $\widetilde{G}(t) \equiv t$; thus this map must be $c_{\mathbf{T}}$-geodesic. The theorem is proved.

\subsection{Additional remarks}

For the bounded functionals $F: \Sigma \rightarrow \mathbb{C}$ with $F(I)=0$, there is a useful lower estimate for $k_{0}(F)$, which allows one to apply Theorem 4.1 effectively. Namely, similarly to [16], one obtains that if $M(F):=$ $\sup _{\Sigma}|F(f)|<\infty$ then the above estimate holds for all

$$
k \leq k_{0}(F)=\frac{\left\|F_{I}^{\prime}\right\|}{\left\|F_{I}^{\prime}\right\|+M(F)+1}
$$

where

$$
\left\|F_{I}^{\prime}\right\|=\frac{1}{\pi} \iint_{\Delta}\left|F_{I}^{\prime}(g(I, z))\right| d x d y
$$

(one has to verify that all arguments in the proof of Theorem 4.1 work well for such $k$ ).

Similar sharp results hold for univalent functions in the unit disk.

For example, the bound (4.4) implies the best known sharp estimates for coefficients of the functions from $\Sigma^{0}$ and $S^{0}$ with dilatations restricted by $(4.11)$.

\section{References}

[1] M. Abate and G. Patrizio, Isometries of the Teichmüller metric // Ann. Scuola Super. Pisa Cl. Sci., 26(4) (1998), 437-452.

[2] S. Dineen, The Schwarz Lemma, Clarendon Press, Oxford, 1989.

[3] C. J. Earle and J. J. Eells, On the differential geometry of Teichmüller spaces // J. Analyse Math., 19 (1967), 35-52.

[4] C. J. Earle and I. Kra, On sections of some holomorphic families of closed Riemann surfaces // Acta Math., 137 (1976), 49-79.

[5] C. J. Earle, I. Kra and S.L. Krushkal, Holomorphic motions and Teichmüller spaces // Trans. Amer. Math. Soc., 944 (1994), 927-948.

[6] C. J. Earle and S. Mitra, Variation of moduli under holomorphic motions, In the Tradition of Ahlfors and Bers (Stony Brook, NY, 1998), Contemp. Math. 256, Amer. Math. Soc., Providence, RI, 2000, pp. 39-67.

[7] F. P. Gardiner and N. Lakic, Quasiconformal Teichmüller Theory, Amer. Math. Soc., 2000. 
[8] H. Grunsky, Koeffizientenbedingungen für schlicht abbildende meromorphe Funktionen // Math. Z., 45 (1939), 29-61.

[9] S. Kobayayshi, Hyperbolic Complex Spaces, Springer, New York, 1998.

[10] S. L. Krushkal, Quasiconformal Mappings and Riemann Surfaces, Wiley, New York, 1979.

[11] S. L. Krushkal, Grunsky coefficient inequalities, Carathéodory metric and extremal quasiconformal mappings // Comment. Math. Helv., 64 (1989), 650-660.

[12] S. L. Krushkal, Extension of conformal mappings and hyperbolic metrics // Siberian Math. J., 30 (1989), 730-744.

[13] S. L. Krushkal, Plurisubharmonic features of the Teichmüller metric // Publications de l'Institut Mathématique-Beograd, Nouvelle série, 75(89) (2004), 119 138.

[14] S. L. Krushkal, Complex geometry of the universal Teichmüller space // Siberian Math. J., 45(4) (2004), 646-668.

[15] S. L. Krushkal, Quasiconformal extensions and reflections, Ch. 11 in: Handbook of Complex Analysis: Geometric Function Theory, Vol. II (R. Kühnau, ed.), Elsevier Science, Amsterdam, 2005, pp. 507-553.

[16] S. L. Krushkal, Extremal problems for Fredholm eigenvalues // Israel J. Math., 172 (2009), 279-307.

[17] S. L. Kruschkal and R. Kühnau, Quasikonforme Abbildungen - neue Methoden und Anwendungen, Teubner-Texte zur Math., Bd. 54, Teubner, Leipzig, 1983.

[18] S. L. Krushkal and R. Kühnau, Quasiconformal reflection coefficient of level lines // Complex Analysis and Dynamical Systems IV, Contemporary Mathematics, 553 (2011), 155-172.

[19] R. Kühnau, Verzerrungssätze und Koeffizientenbedingungen vom Grunskyschen Typ für quasikonforme Abbildungen // Math. Nachr. 48 (1971), 77-105.

[20] R. Kühnau, Wann sind die Grunskyschen Koeffizientenbedingungen hinreichend für Q-quasikonforme Fortsetzbarkeit? // Comment. Math. Helv., 61 (1986), 290307.

[21] D. Minda, The strong form of Ahlfors' lemma // Rocky Mountain J. Math., 17 (1987), 457-461.

[22] Chr. Pommerenke, Univalent Functions, Vandenhoeck \& Ruprecht, Göttingen, 1975.

[23] E. Reich and K. Strebel, Extremal quasiconformal mappings with given boundary values, Contribution to Analysis (L.V. Ahlfors et al., eds.), Academic Press, New York, 1974, pp. 375-392.

[24] H. L. Royden, Automorphisms and isometries of Teichmüller space, Advances in the Theory of Riemann Surfaces (Ann. of Math. Stud. vol. 66), Princeton Univ. Press, Princeton, 1971, pp. 369-383.

[25] G. Schober, Univalent Functions - Selected Topics, Lecture Notes in Math. 478, Springer, Berlin, 1975.

[26] Y. L. Shen, Pull-back operators by quasisymmetric functions and invariant metrics on Teichmüller spaces // Complex Variables, 42 (2000), 289-307.

[27] K. Strebel, On the existence of extremal Teichmueller mappings // J. Analyse Math., 30 (1976), 464-480. 
[28] E. Vesentini, Complex geodesics and holomorphic mappings // Sympos. Math., 26 (1982), 211-230.

[29] I. V. Zhuravlev, Univalent functions and Teichmüller spaces // Inst. of Mathematics, Novosibirsk, preprint, (1979) 1-23 (Russian).

\section{CONTACT INFORMATION}

Samuel L. Krushkal Department of Mathematics, Bar-Ilan University 52900 Ramat-Gan, Israel

Department of Mathematics, University of Virginia, Charlottesville, VA 22904-4137, USA

E-Mail: krushkal@math.biu.ac.il, krushkal@macs.biu.ac.il, slk6z@virginia.edu 